\title{
Correlated, Real Time Multi-spectral Sensor Test and Evaluation (T\&E) in an Installed Systems Test Facility (ISTF) Using High Performance Computing
}

\author{
John Kriz ${ }^{1}$, Tom Joyner ${ }^{1}$, Ted Wilson ${ }^{1}$, and Greg McGraner ${ }^{1}$ \\ ${ }^{1}$ Naval Air Warfare Center - Aircraft Division (NAWC-AD), Air Combat Environment \\ Test \& Evaluation Facility (ACETEF), Building 2109, Suite N223, Shaw Road, Patuxent River, \\ MD 20670 \{KrizJE, JoynerTW, WilsonJE2, McgranerGL\} @ navair.navy.mil
}

\begin{abstract}
Military aircraft are increasingly dependent on the use of installed RF and Electro-optical sensor systems and their data correlation/fusion to contend with the increasing demands and complexities of multi-mission air warfare. Therefore, simultaneous, and correlated, ground-based T\&E of multiple installed RF and Electro-optical (EO) sensors and avionics systems/subsystems is used to optimize the use of scarce flight testing resources while also satisfying increasingly sophisticated test requirements. Accordingly, it is now an accepted, and highly costeffective practice, to first conduct rigorous ground testing and evaluation (T\&E) of the installed/integrated sensors' performance and their interaction using high fidelity modeling and simulation with real time scene rendering. Accordingly, valid groundbased $T \& E$ of installed multi-spectral (RF, IR, Visible) sensors requires that the simulations' spatial, spectral and temporal components be of sufficient fidelity and correlation to produce sensor responses that are indistinguishable from responses to "real-world" conditions. This paper discusses accomplishment of the foregoing goals, and fidelity challenges through innovative utilization of High Performance Computing (HPC) parallelization, visualization, shared memory optimization and scalability for multi-spectral modeling, simulation and real time rendering.
\end{abstract}

\section{Introduction}

Today's military aircraft are increasingly dependent on a diversity of electromagnetic sensors to provide aircrews and tactical commanders with unparalleled battle space awareness and engagement opportunity. The missions are equally diverse in such areas as reconnaissance, search and rescue, time vision navigation/evasion, target acquisition, target search and track, missile warning, and terminal missile homing. Achieving these missions requires employment of the most advanced sensors available to provide the war fighter with the greatest possible tactical advantage. Accordingly, multi-spectral sensors are an integral component of the flight and mission control avionics. Consequently, overall mission performance is directly linked to the combined performance of the onboard mission critical sensors. 


\subsection{Installed Sensor Testing}

The foregoing critical inter-relationship necessitates extensive developmental and operational sensor test and evaluation (T\&E) of these integrated sensor/avionics environments prior to their release for operational use. The highest fidelity of systems performance testing occurs during flight-testing. However, it is becoming increasingly cost prohibitive to provide all mission representative environments during flight-testing. Therefore, the aviation community has developed ground-based dynamic, high fidelity instrumentation capabilities to test installed aircraft sensor subsystems. These ground-based capabilities are increasingly using correlated high fidelity real-time simulation and stimulation to conduct T\&E of the installed sensor's performance and interaction with other sensors integrated within the weapons system platform. The computational challenge is to replicate the multi-spectral, temporal and geometric fidelity of the real-world problem-domain in a virtual warfare environment. Figure 1 illustrates the many attributes to be replicated, and correlated in real-time.

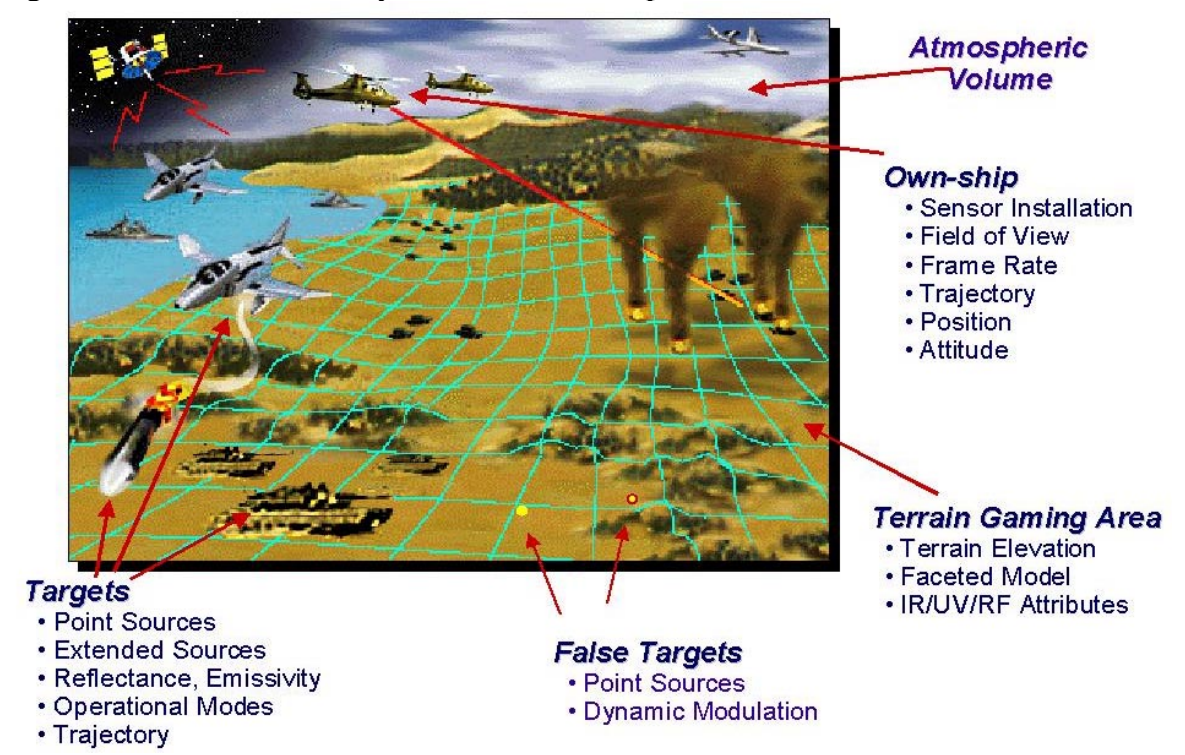

Fig. 1. The Computational Challenge: Accurately Replicate the Battle-space

\subsection{Joint Installed System Test Facility (JISTF)}

The combination of increasingly sophisticated test requirements and today's declining investments in test assets requires the coordinated and innovative development of modeling, simulation and emerging technologies to maximize use of scarce testing investment resources. In response to these emerging T\&E needs, the Office of the Secretary of Defense (OSD), Central Test and Evaluation Investment Program (CTEIP), has sponsored development of three Joint Installed System Test Facility (JISTF) enhancements based on dynamic virtual reality simulation technology. 
These enhancement projects are the Communications Navigation \& Identification (CNI)/Joint Communications Simulator (JCS), Generic Radar Target Generator (GRTG), and Infrared Sensor Stimulator (IRSS). This paper addresses the capabilities of the JISTF GRTG and IRSS enhancements at the Air Combat Environment Test and Evaluation Facility (ACETEF), Naval Air Warfare Center, Aircraft Division (NAWC-AD), Patuxent River, MD, and their HPC requirements, and utilization. GRTG and IRSS provide the capability to simultaneously test multiple installed avionics and sensor subsystems in a ground test environment. Moreover, they are used to evaluate multi-sensor data fusion/correlation and subsystems' interoperability for Communications and Data Link subsystems, GPS, RADAR, and Infrared Sensors. Figure 2 illustrates the functional relationship of the three upgrades within the ACETEF architecture.

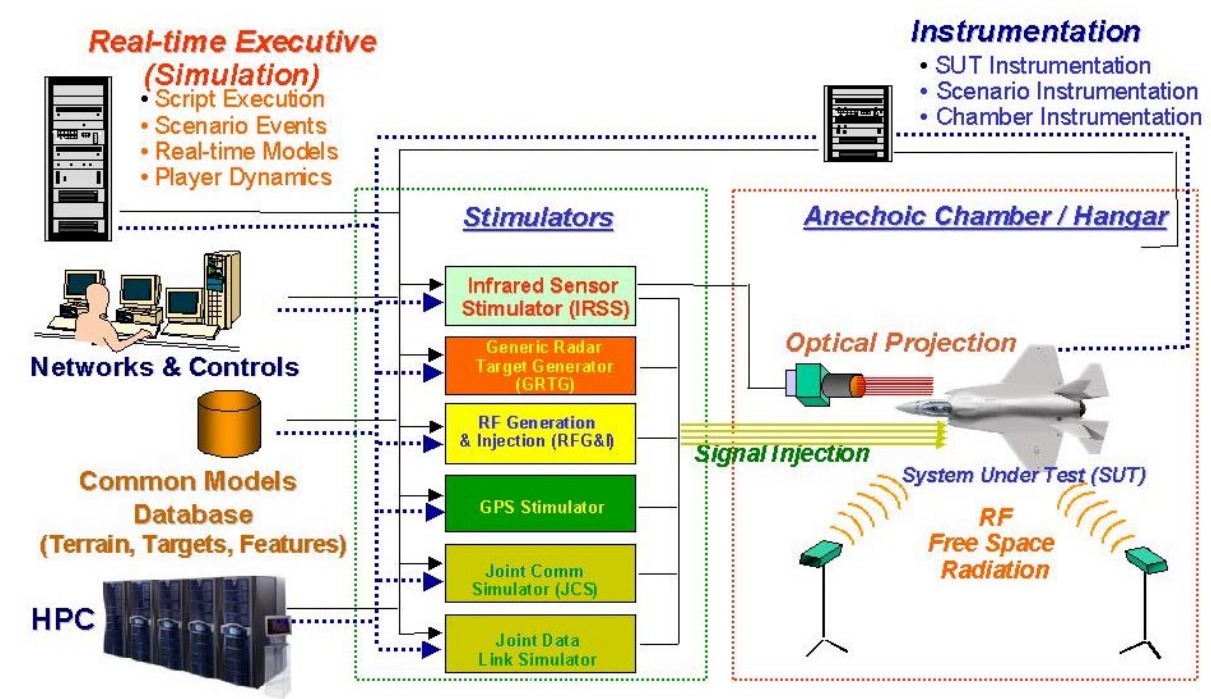

Fig. 2. Functional Block Diagram of Upgrades in ACETEF Architecture

\subsection{Overview of the ACETEF Enhancements}

The objective of the ACETEF enhancements is to develop, install, and operationally certify common core capability enhancements that will enable the effective testing of advanced CNI, RADAR, Infrared (IR) and Electronic Countermeasures (ECM) avionics subsystems. This enhanced test capability reduces the overall cost and schedule associated with the developmental and operational testing of existing and new avionics, sensor, and communication suites. 


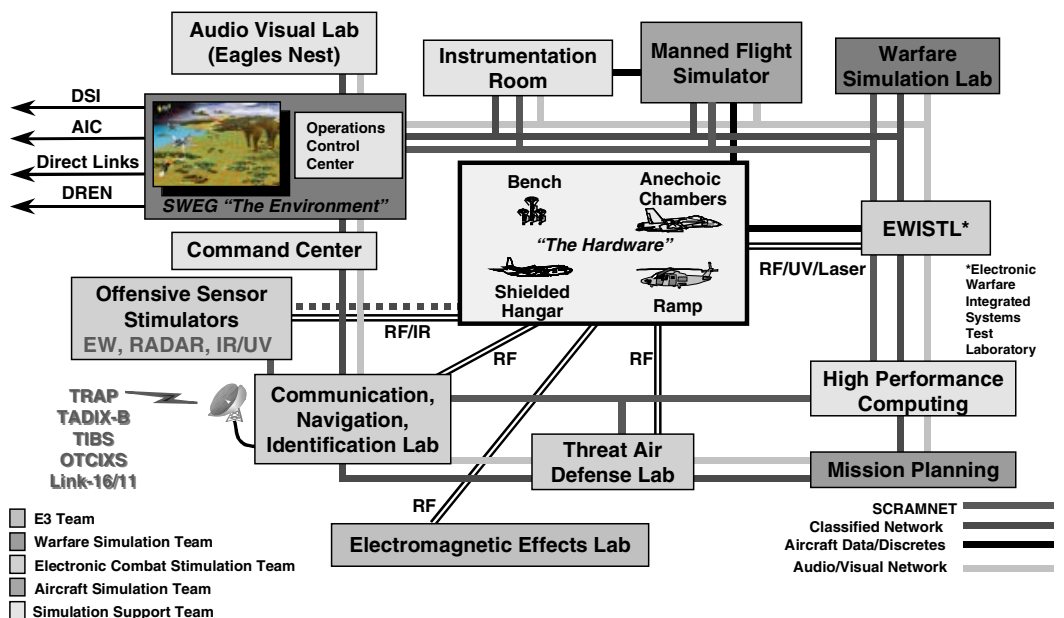

Fig. 3. Functional Architecture of ACETEF Infrastructure

This new capability supplements conventional flight testing by enabling the assessment and evaluation of system and subsystem performance over a wide range of operational modes, threat scenarios, and Electronic Warfare (EW) environments both prior to and during flight-testing. These assessments greatly increase the information value and content of each flight test by focusing attention on specific preparatory high value tests that are best be performed in the air. In addition, these assessments provide the capability to conduct in-depth evaluations of flight test anomalies in an installed configuration. The ACETEF infrastructure illustrated in Figure 3 includes two anechoic chambers, a shielded hangar, associated test instrumentation, HPC resources and laboratories capable of testing installed sensor/avionics systems.

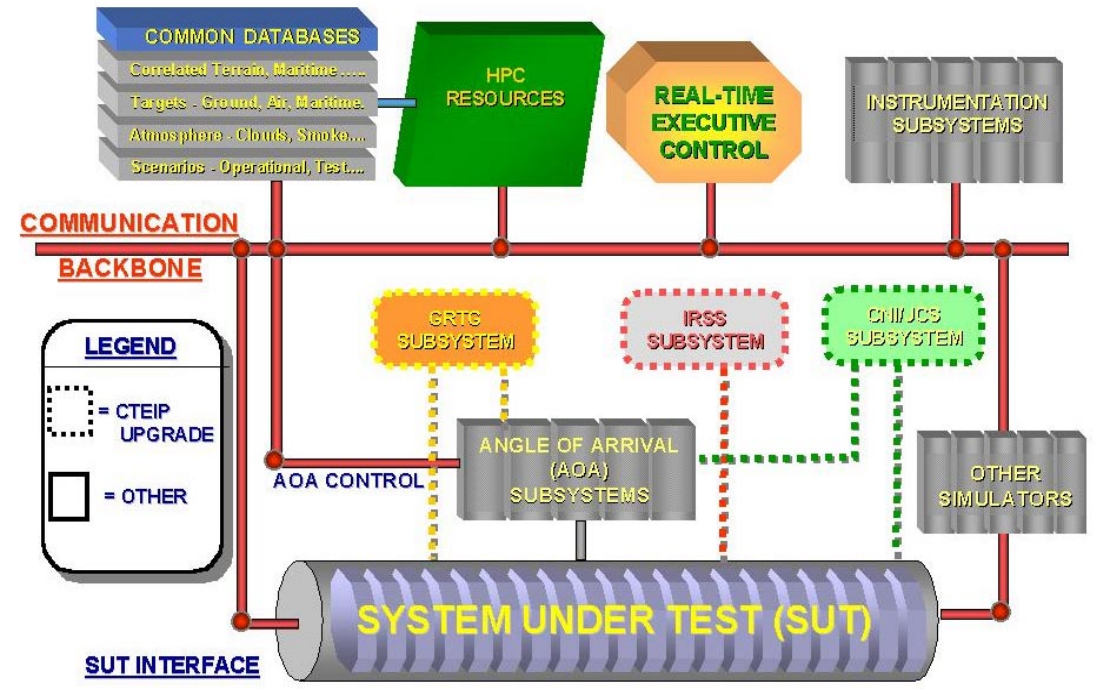

Fig. 4. Functional Block Diagram of ACETEF Upgrade Interoperability 
Figure 4 illustrates the integration and interoperability of the GRTG and IRSS within the ACETEF infrastructure. Interoperability is achieved through a common communications backbone, which enables the real-time executive (RTEx) control to synchronize individual simulator/stimulator operations, the System Under Test (SUT) and the Instrumentation Subsystem to a common test scenario. Common data bases, standard communications interfaces, standard programming languages, and standard simulation models all contribute to the integrated operation of the ACETEF. Modular design principles and the use of an open architecture permit the addition of other simulators and a free space Angle Of Arrival (AOA) system in the future. The communications infrastructure also implements a number of standard interfaces to avionics buses through a SUT interface. The use of common test scenarios, common data bases and standard simulations used by hardware-in-the-loop (HITL) facilities and Open Air Ranges (OAR) provides the linkage and traceability needed to implement the Electronic Combat (EC) Test Process.

Additionally, their capabilities contribute to the evaluation of systems, and sensors, during development, and engineering model development (EMD) performance assessment. They also enable sensor test in a variety of environmental and warfare simulations that can not be replicated on the test range.

\subsection{Generic Radar Target Generator (GRTG)}

The GRTG is a modular and expandable radar environment stimulator for use with existing and emerging radar systems (e.g., Existing - F-22 APG-77, F/A-18 APG-73, and the JSF - Future capability). It is used to verify the functionality of air-to-air (A/A) and air-to-surface (A/S) modes (- Future capability) of radar systems. The GRTG generates radar skin returns and surface returns, and electronic countermeasures (ECM) against airborne radar's operating in the I/J bands (8$18 \mathrm{GHz}$ ). It is capable of simulating a maximum of four targets in the main beam of the radar antenna. The modular design enables reconfiguration to incorporate enhancements in other spectra bands and radar modes as necessary to accommodate future systems. Additionally, 32 targets can be simulated in the radar field of regard (FOR).

The GRTG can be operated in a stand-alone mode or integrated with the ACETEF infrastructure. Signals are injected directly to the SUT radar receiver via RF cables or radiated free space from horn antennas to the radar antenna assembly. In the case of free space, the GRTG functions in conjunction with the angle of arrival (AOA) subsystem to provide multiple dynamic/static angles of arrival for radar/ECM targets. In the case of direct injection (RF), the GRTG replicates/models by-passed radar subassemblies by directly injecting the signal into the radar-processing avionics. The GRTG is capable of both autonomous (stand-alone) testing and correlated, real time, integrated multi-spectral testing with other simulators/stimulators (e.g., EW, IR, GPS). The latter form of testing is accomplished with the GRTG and other simulators/stimulators responding to an external ACETEF scenario simulation synchronized by the real-time executive controller. Figure 5 is a block diagram of the GRTG functional areas and relationships. 


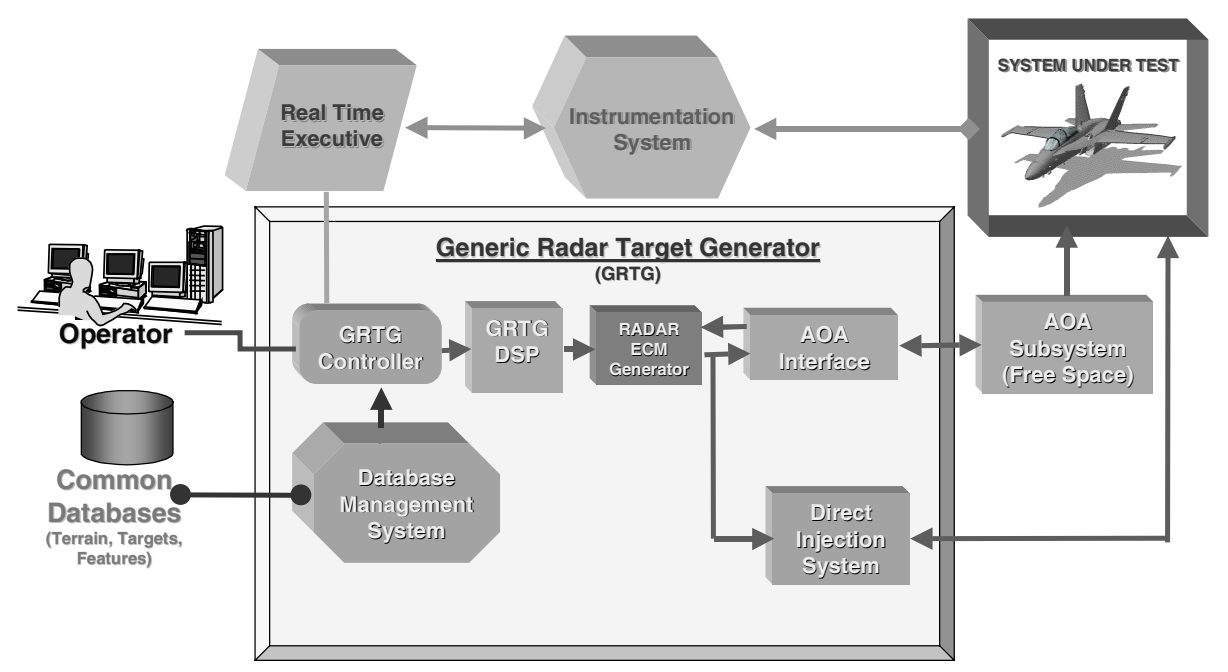

Fig. 5. GRTG Functional Block Diagram

\subsection{Infrared Sensor Stimulator (IRSS)}

The IRSS is a modular system used to generate high fidelity Infrared (IR) scenes for stimulation of installed IR Electro-Optic (EO) sensors installed on military aircraft undergoing integrated developmental and operational testing. It is capable of stimulating multiple types of sensors such as Forward Looking Infrared (FLIR), Missile Warning Systems (MWS), Infrared Search and Track (IRST) and Missile Seekers. It features include an open architecture, high fidelity physics-based rendering, repeatable, dynamic real-time IR and Ultraviolet (UV) simulation, freespace scene projection and direct image signal injection stimulation capability. The dedicated system configuration utilizes two SGI Onyx2 graphics computers and a proprietary scene rendering system (SRS) to provide three channels of real time sensor-specific scene simulation. With the parallel use of HPC resources, IRSS is scalable to ten channels of simulation output. The signal injection component also provides a sensor modeling capability for replicating sensor optics/detector modulation transfer function and evaluating performance characteristics and effectiveness of prototype designs (e.g., EMD). The IRSS system can operate in stand-alone mode, or in an integrated mode with the ACETEF infrastructure.

Figure 6 is a top-level functional block diagram of the IRSS and its six subsystems. The six subsystems are (1) Simulation Control Subsystem; (2) Scenario Development Subsystem; (3) Modeling and Database Development Subsystem; (4) Scene Generation Subsystem; (5) Point Source Projection Subsystem and (6) Signal Injection Subsystem/interface. The IRSS operator and/or the real-time executive control software provide inputs to the IRSS simulation control computer. 
In the integrated mode, the ACETEF real time executive software controls IRSS SCRAMNET interfaces, timing, and execution priorities for system models and simulations to create a real-time virtual test environment. Scenario development inputs are provided by an operator and/or the executive software. Inputs includes (but are not limited to): aircraft data (position, airspeed, attitude), sensor data (FOV, FOR, modulation transfer function (MTF), and slew rates), and gaming area environment (targets, backgrounds and atmospherics effects). The IRSS functions in concert with the other stimulators to perform correlated, real time, integrated multi-sensor testing.

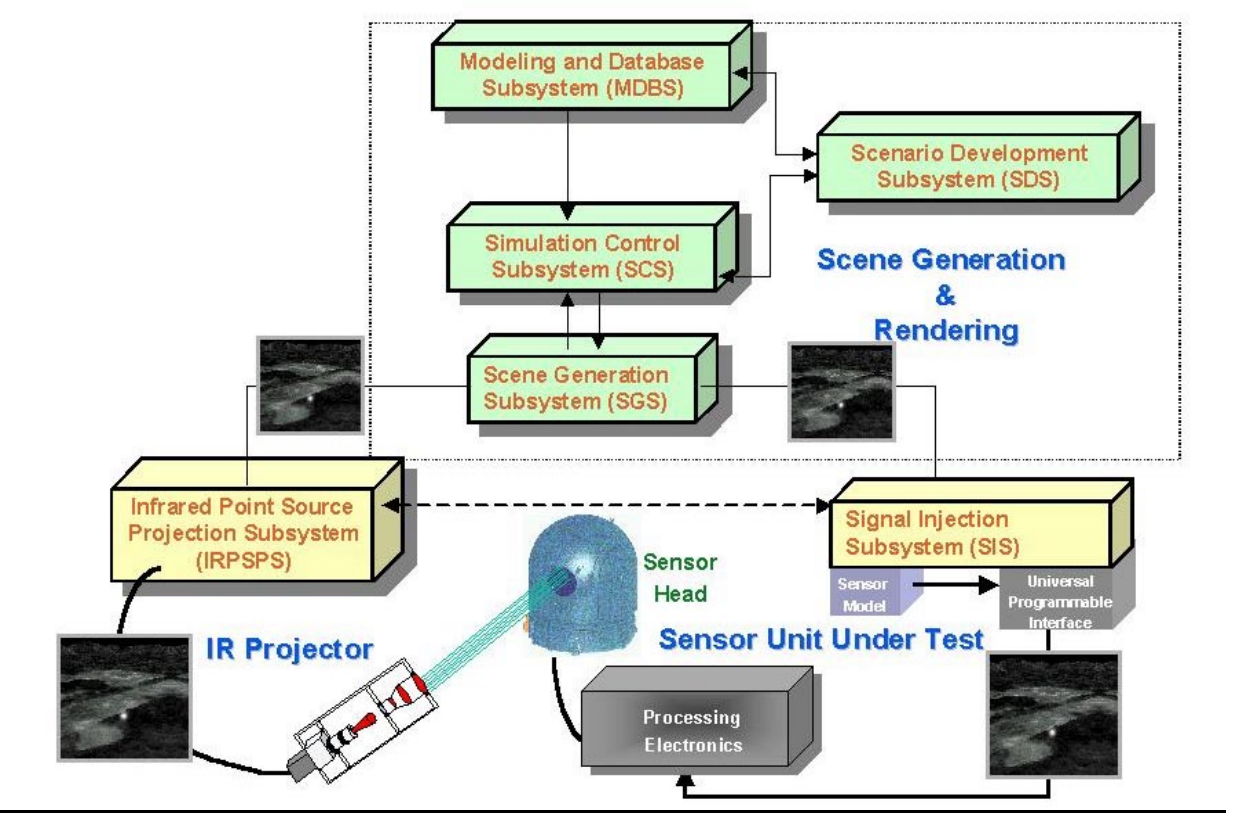

Fig. 4. IRSS Six Primary Functional Subsystems

\section{The Need for High Performance Computing (HPC) Resources}

The technical challenge for the ACETEF T\&E enhancements is that they be valid test tools. Consequently, the spatial, spectral and temporal components of the computergenerated synthetic scenes/RF responses should be of sufficient fidelity to produce sensor responses that are indistinguishable from the tested sensor's "real-world" responses. Two fundamental capabilities are required to accomplish the foregoing validity objectives. First, the simulation generation equipment must have the capability to create a valid simulation of the real-world conditions. Second, the stimulators (RF emitters, IR scene projectors and signal injection modules) must be capable of accepting the simulated scene, retain sufficient scene fidelity, and interface appropriately to the sensor unit under test (UUT). 
This section discusses the subtleties of accomplishing the foregoing goals, and provides an overview of how these $\mathrm{T} \& \mathrm{E}$ enhancements meet the fidelity challenge. The GRTG and IRSS have been designed to take advantage of present and future ACETEF HPC resources. When the complexity of GRTG and IRSS test requirements increases, HPC resources are used to augment dedicated computational systems. Areas such as increased number and/or types of sensors to be stimulated, increased frame rates, and scene complexity are illustrative of increased test requirements. Figure 7 is an architectural diagram of the ACETEF HPC configuration.

\section{ACETEF High Performance Computing}

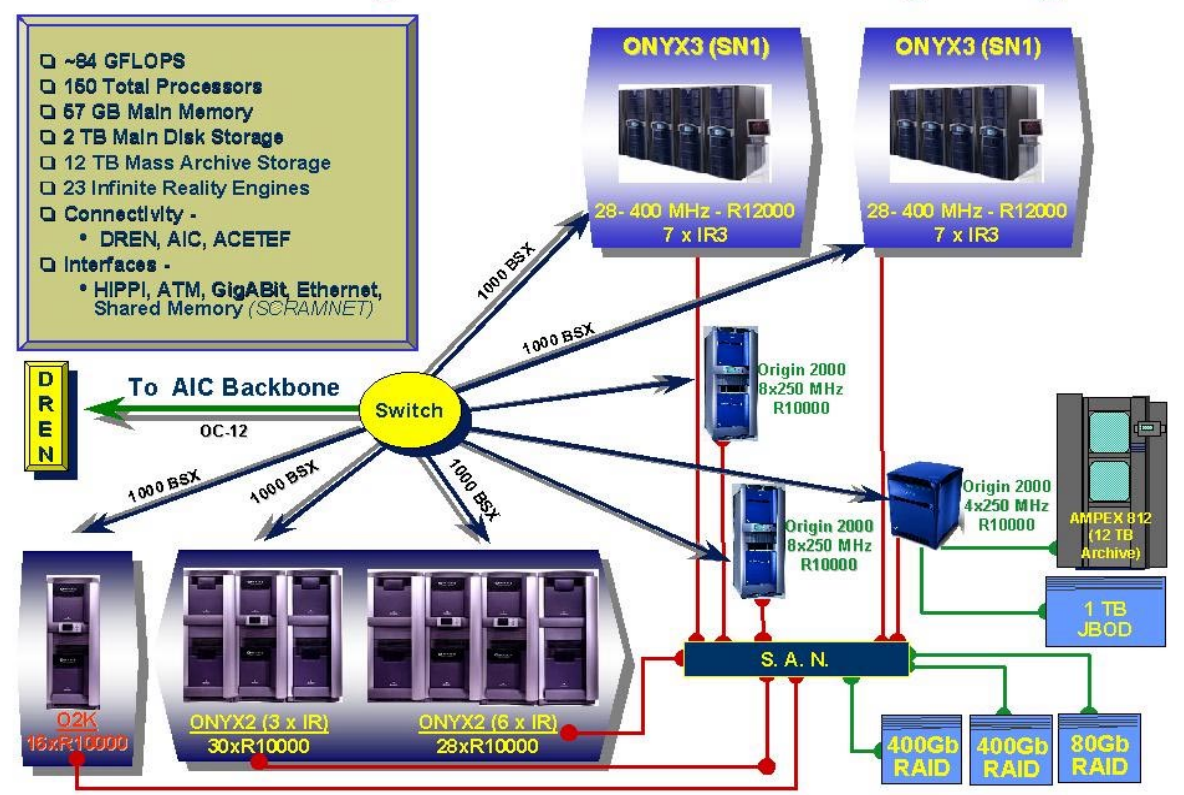

Fig. 7. ACETEF High Performance Computing Architectural Configuration

The GRTG configuration is capable of utilizing HPC resources for real-time standalone scenario control. Pre-planned product improvements to the GRTG includes a large area common terrain database server upgrade to support ultra-high-fidelity synthetic aperture radar (SAR) processing. The terrain database will be shared between the IRSS, and the GRTG to support multi-spectral testing. Figure 8 is an aerial view of the ACETEF Large Anechoic Chamber with a cutaway view of an F/A 18 and EA6B inside the chamber. Figure 9 illustrates an ACETEF multi-spectral testing configuration in one of the two anechoic chambers. The aircraft can be positioned on the ground or suspended from one of two 40 tons cranes located on the test chamber ceiling. 


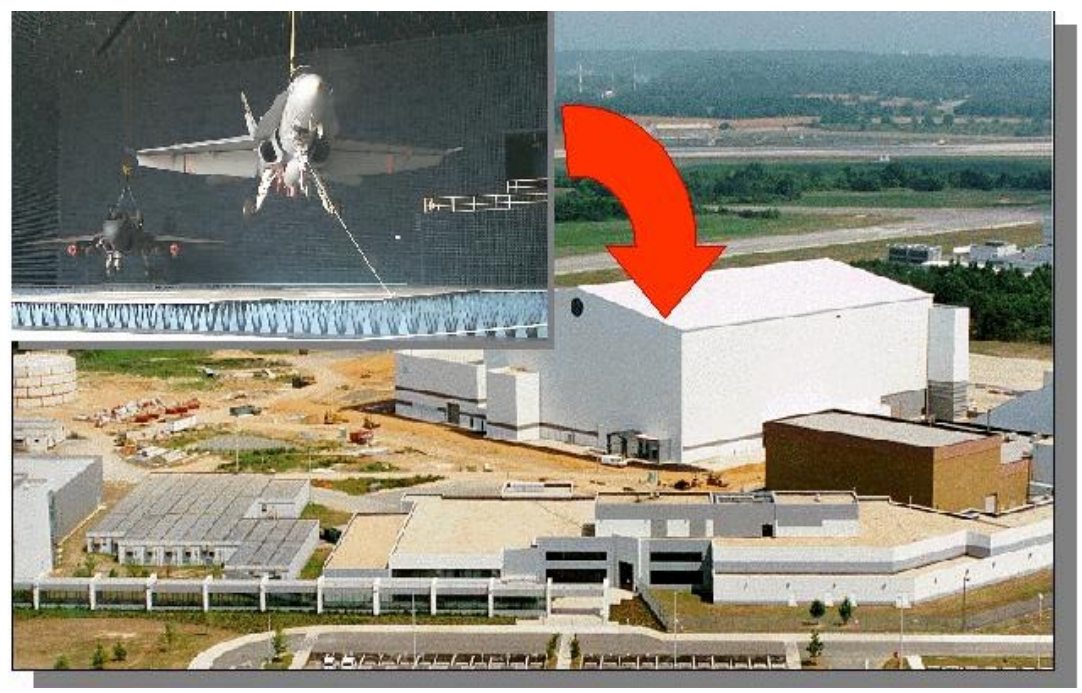

Fig. 8. ACETEF Large Anechoic Chamber
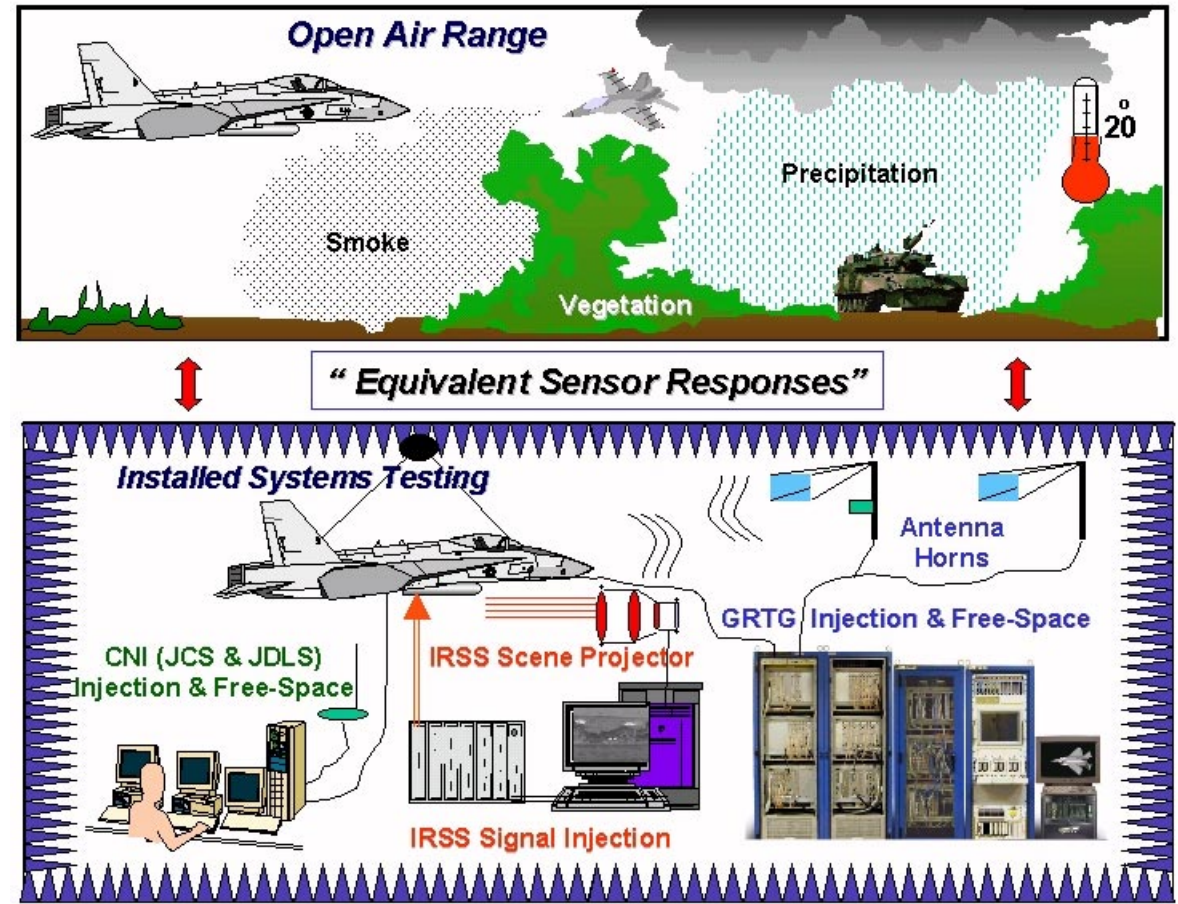

Fig. 9. ACETEF Multi-Spectral Testing Configuration 
The IRSS performance and throughput requirements for dynamic real time rendering and physics-based accuracy necessitate use of an HPC solution. Accordingly, the IRSS architecture employs commercial-off-the-shelf (COTS) Silicon Graphics (SGI) fast symmetric multiprocessing hardware to minimize cost and development time. The following functional processes illustrate the high performance computational workload that the IRSS must satisfy.

1. IRSS provides three channels of digital video configured to support synchronized testing of multiple EO imaging sensors on a single aircraft or individual sensors on up to three aircraft. The architecture is scalable to a maximum of ten channels of output using a commensurate temporary allocation of HPC resources.

2. During real-time scene simulation, the multiprocessors are used to update polygon vertex locations and compute radiometrically correct floating-point radiance values for each waveband. Scene radiance is calculated on a frame by frame basis with each frame being rendered with the relevant contributions from the sky, sun, targets, terrain, and atmosphere. The individual frame calculations are accomplished as a function of the engagement geometry using existing validated high-fidelity IR models for phenomenology, terrain, and targets.

\section{Summary and Conclusions}

The JISTF IRSS and GRTG enhancements have all been carefully designed and developed to leverage current and future HPC resources. The IRSS enhancement is increasingly using more of HPC assets as it pushes the limits of technology for IR scene generation and rendering. When the GRTG and the CNI systems are upgraded to take advantage of a multi-spectral common terrain database systems, there will be increased reliance on HPC systems to provide the backbone for integrated multispectral testing at ACETEF. They provide the valid stimulator T\&E of installed multi-spectral (RF, IR, Visible) sensors with correlated high fidelity stimulation spatial, spectral and temporal components that produce sensor responses that are indistinguishable from responses to "real-world" conditions. The availability and use of HPC resources has enabled resolution of critical issues relating to scaling, and the accommodation of additional channels of synchronized/correlated output and throughput limitations versus the level of scenario complexity (e.g. the content of targets and background descriptions, and system latency.)

\section{References}

1. T. Joyner, K. Thiem, R. Robinson, R. Makar, and R. Kinzly, "Joint Navy and Air Force Infrared Sensor Stimulator (IRSS) Program Installed Systems Test Facilities (ISTF)", Technologies for Synthetic Environments: Hardware-in-the-Loop Testing IV, Robert Lee Murrer, Editor, Proceedings of SPIE Vol. 3697, pp. 11-22, 1999.

2. DoD HPC Modernization Program FY96 Implementation Plan for the Common High Performance Computing (HPC) Software Support Initiative (CHSSI), dated October 1995 